\title{
RESEARCH ON AUTO MOBILE-PC UPLOAD IMAGES APPLICATION THROUGH BLUETOOTH USING JAVA
}

\author{
SALMAN $^{[1]}$, HASHIM ALI $^{[2]}$, SALMAN SALEEM $^{[3]}$ \\ ${ }^{1,2}$ Department of Computer Science, Abdul Wali Khan, University Mardan, Khyber \\ Pakhtunkhwa, Pakistan \\ ${ }^{3}$ Department of Telecommunication Engineering, UET Peshawar, \\ Khyber Pukhtoonkhwa, Pakistan \\ Email: ${ }^{[1]}$ salman@awkum.edu.pk \\ ${ }^{[2]}$ hashimali@awkum.edu.pk \\ ${ }^{[3]}$ engrsalmansaleem@gmail.com
}

Revised October 2015

\begin{abstract}
The popularity of java programming is growing continuously, as Java gives an independent, object oriented and multithreaded programming environment.[1] J2ME (Java 2 Micro Edition) is the Most Ubiquitous Application Platform for Mobile Devices and provides a robust flexible environment for all application running on cell phones and also other embedded devices [11][12]. In this paper an application is develop for mobile phone and PC (Personal Computer), using Java programming language. The main function of this application is to transfer images automatically from mobile phone to PC or laptop and also save these images in computer system memory (Hard Disk), whenever mobile phone detect PC or laptop in its Bluetooth range. The mobile phone app save the PC name in its database as server and sends the images to only that $P C$ while the rest of the $P C$ in its Bluetooth range are ignored plus the mobile app also save the sent images name list so that the images are not send twice to PC. This application has been tested on student's mobile phones in the university premises and shows a promising results and will also be tested on public mobile devices in future studies.
\end{abstract}

Keywords: Mobile Computing; J2ME (Java 2 Micro Edition); J2SE (Java 2 Secondary Edition); Bluetooth.

1. Introduction. Wireless technology is succeeding a great importance around the world, normally people appreciate the wireless technologies rather than cables, as a result the wireless technologies are intending to replace cables connection between mobile phones (cell phones), PDAs (personal digital assistant) and also other portable devices[14][3]. In the present scenario, if wireless communication becomes unavailable then the world may be virtually stops because the global economy and our life style are completely dependent on the flow of information. The best example is internet, TV, radio and mobile phones as mobile phones have become highly available during the last ten years [3]. Now days approximately every one in this world has mobile phone, which make them available no matter where they are. During the last few years the rate of new wireless technologies are increasing day by day. M. S. Gast (802.11 Wireless Networks) [6] publish that In the last few years IEEE802.11 technologies spread so much rapidly that even the people or consumers setup their own wireless networks. This shows that large organizations are not the only one to provide the wireless networks but the individuals can also implement wireless networks as well. As new areas in wireless networks are introduced, nearly about every single individual in the world is dependent on wireless communication or 
wireless networks.[15][14].

Bluetooth technology is also a part of wireless technology or wireless networks and also spreading rapidly per year. Everyone is highly dependent on mobile phones or cell phones because it is a general need of every one and we know that Bluetooth wireless technology is also available in all new mobile phones (cell phones). According to http://www.mobilemag.com,[16] in 2003 the Bluetooth chipsets are double then the year 2002 that is 69 million Bluetooth chipsets are shipped and maximum of them are used in cell phones (mobile phones). Bluetooth PANs (Personal Area Networks), through which all Bluetooth devices can communicate with one another are free because Bluetooth technology is a low cost, short range radio and also low power wireless technology[2][9]. Bluetooth technology also intended to replace the cables and wires that will helps to clean up your desk from cables and wire between your mouse, laptop, workstation or pc computers and other portable devices.[1]

In this paper, two separate software applications named as Client Bluetooth Software for mobile phone and Server Bluetooth Software for PC or laptop is designed. Client Bluetooth Software search images in the mobile phone's memory and in its memory card and sends it automatically to Server Bluetooth Software once it is found in the range of Bluetooth. When Server Bluetooth Software receives these images from Client Bluetooth Software it stores them in the computer memory (secondary memory or hard disk). As this software automatically uploads images to PC from mobile phone, so Client Bluetooth Software checks all the images to avoid the copy of images in the sending process.

Different tools have been used to develop the software which includes J2ME (Java 2 Micro Edition) to design the mobile software (client Bluetooth software) and J2SE (Java 2 Secondary Edition) for computer system software (server Bluetooth software)[9][10][11].

2. Auto Mobile-PC Block Diagram: The figure 1, below shows the auto transfer of images from mobile phone to computer system; when mobile phone (Client Bluetooth Software) detects the computer system or laptop (Server Bluetooth Software) in the range of Bluetooth. If mobile phone app found some other Bluetooth devices in its range it will be ignored as mobile phone app store the PC name in its database as server name. Once the PC app received the images from mobile phone, it will store them automatically in the computer memory (Hard Disk).

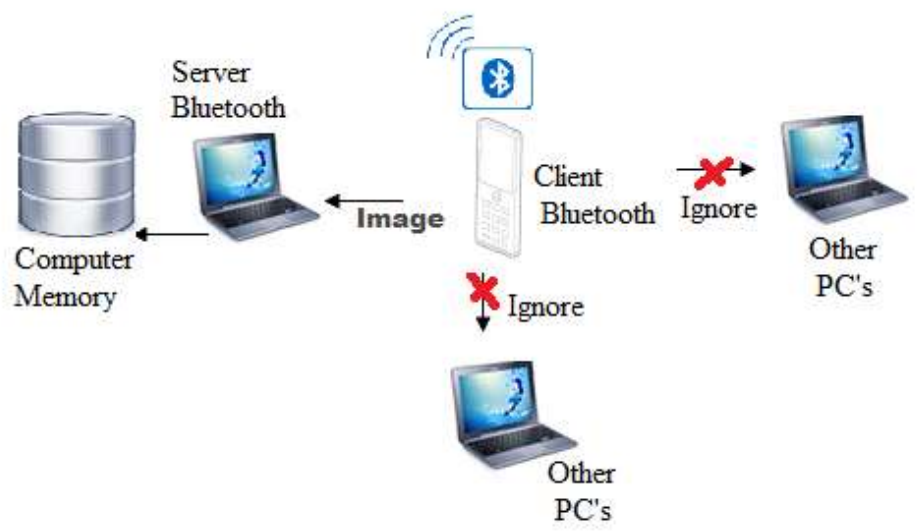

Figure 1: Block Diagram of Auto Mobile to PC

3. Results and Discussions: As we know that two separate applications named as Client Bluetooth software and Server Bluetooth software are working together to archive the task (transfer images automatically from mobile phone to $\mathrm{PC}$ once the mobile phone find the $\mathrm{PC}$ in it Bluetooth range). Client Bluetooth software explains the whole functionality of mobile phone app while Server Bluetooth software demonstrates the overall functionality of PC app both of them are discussed below. 
A. Client Bluetooth Software: The client Bluetooth software once installed in the mobile phone. it start searches the images in the mobile phone memory and in the mobile phone memory card. When the client Bluetooth software is running for the first time it displays the message of set server first (as the server name is not found in the client Bluetooth software database) shown in the figure 2. Select the Set option from menu, it automatically start searching the nearby Bluetooth devices and display the list of Bluetooth devices as shown in the figure 2; select the sever PC in which the Server Bluetooth software is install as shown below.
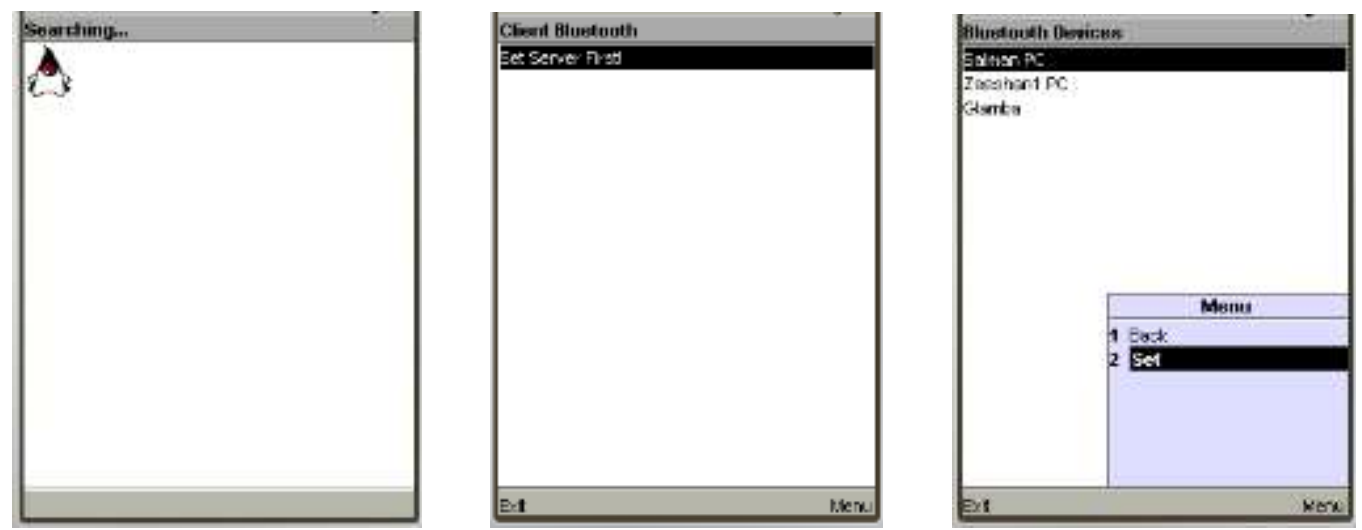

Figure 2: Searching server, Set Server First \& Set Server Menu Options

When the set option is used the client Bluetooth software will save the name of the Bluetooth device in the database, so that, for the next time no need to search the server Bluetooth, client Bluetooth software will automatically read it from database and start sending images.

Every time the Client Bluetooth software search the images in the mobile phone if found, it will check the images with previously sent images list, so that no image is repeated or send twice to server Bluetooth software (PC). Once the searching and checking images stage is completed, the client Bluetooth software has a list of new images, that would be send to server Bluetooth (PC or Laptop). Now the client Bluetooth software continuously searching the server using Bluetooth and when it found that the server is in the range of Bluetooth it automatically start sending images to server Bluetooth (PC or Laptop). Once client Bluetooth software sends all the images to server Bluetooth software, it displays the successful message, as shown in the figure 3 .
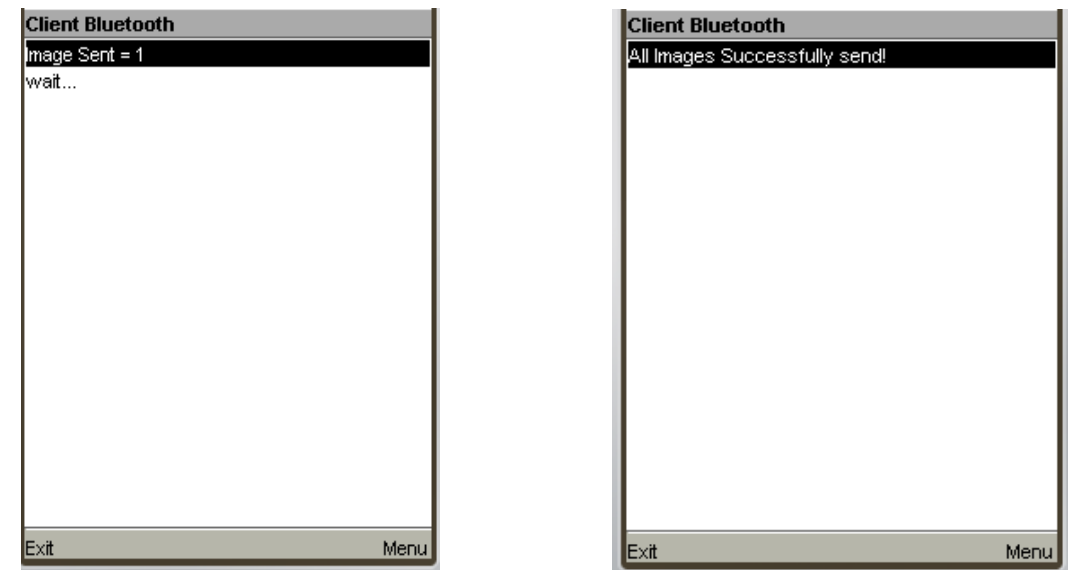

Figure: 3 Sending images to PC \& Confirmation of Images

B. Server Bluetooth Software: When the server Bluetooth software starts, it displays the GUI (Graphical User Interface), as shown in the figure 4. Select the start button to start the server Bluetooth software. 


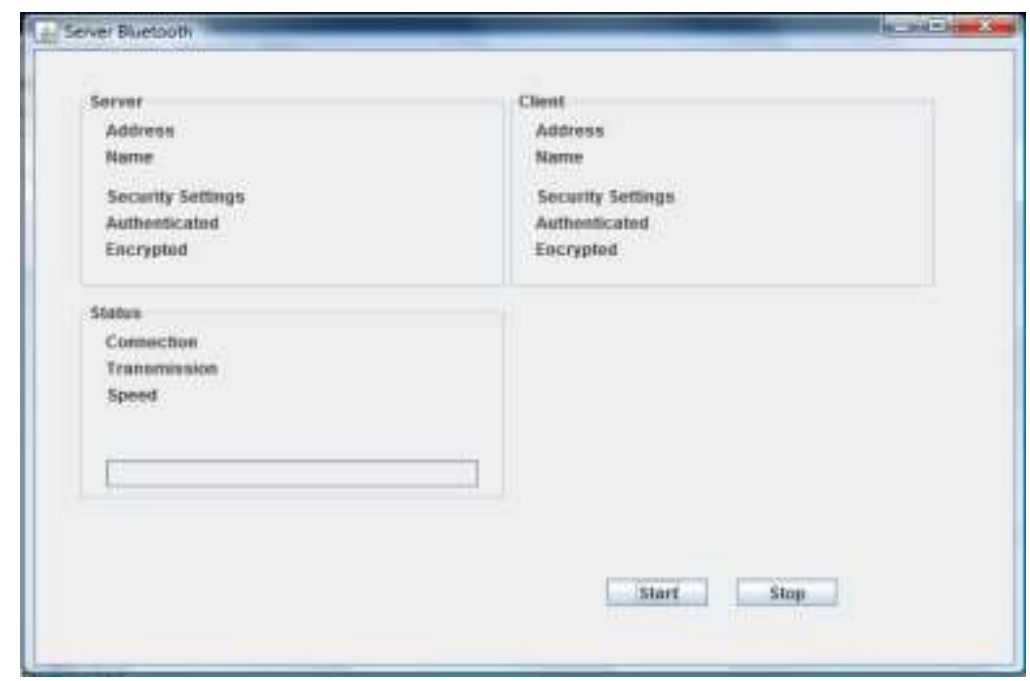

Figure 4: Server Bluetooth App

Once the software starts it initialize the local Bluetooth and display Bluetooth name, address, security setting, authentication and encryption and start waiting for client Bluetooth software to send images, as shown in the figure 5 .

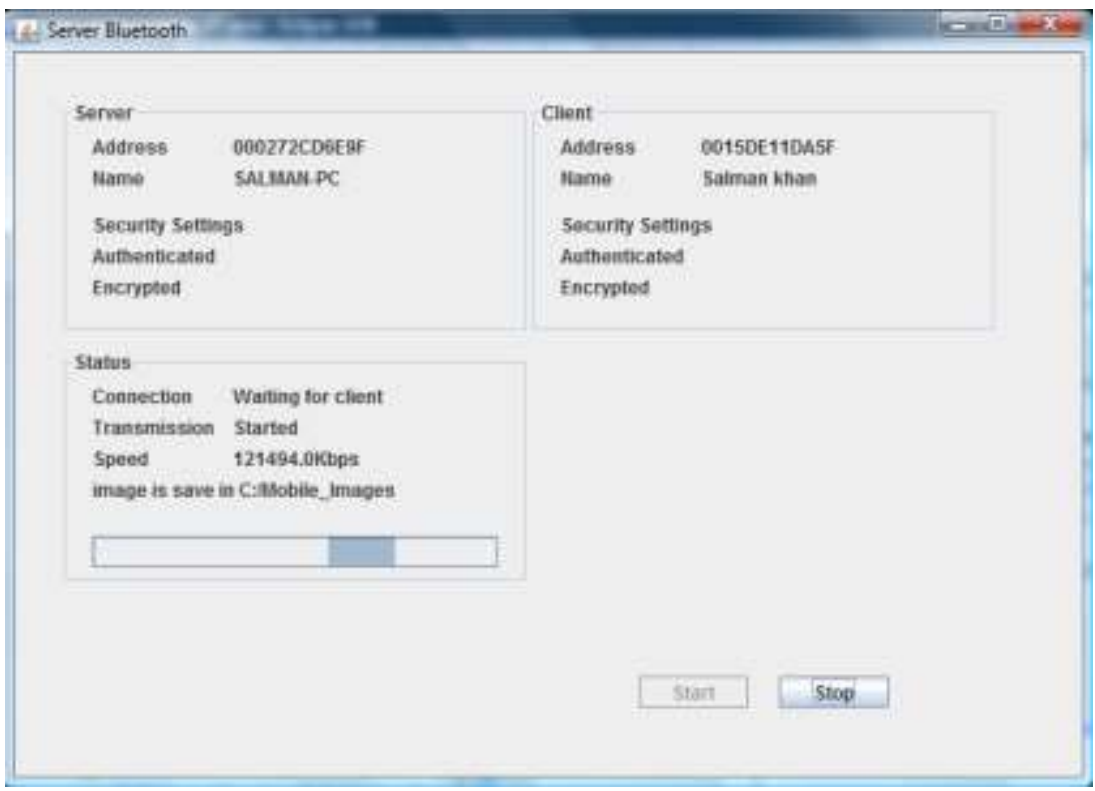

Figure 5: Receiving Images from Mobile Phone (Client Bluetooth Software)

Once it is connected with client (Bluetooth software), it automatically receive the image(s) file and save it directly in the PC memory as shown in the figure 6. Then it display some important information like speed KBPS, image save destination and also display the client Bluetooth information in the client portion like name of the client Bluetooth device, address and also some other information like security settings enable or disable, or null authenticated yes or no, or null and also encrypted or not. Once it receive image, Server Bluetooth software start again waiting for the next image, until the last image. 

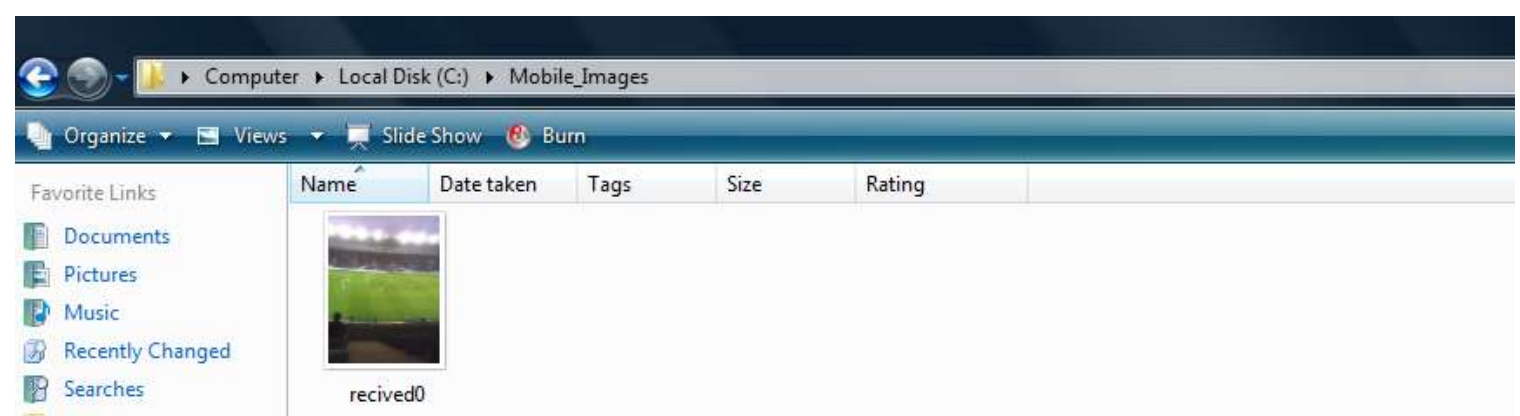

Figure 6: Save auto Image(s) in Computer Memory

In the client Bluetooth software there are also some other extra facilities like set server, which can be used to save the server (PC or Laptop) name in it. Therefore, there would be no need to search repeatedly the serve once it is already set or save in the client software database. This set server information is also editable, so that end user can change the server Bluetooth any time they want. Client Bluetooth software has also the facility to show the list of images already sent to server Bluetooth software (PC or Laptop) from client Bluetooth software (mobile phone) and also this sent images list can be reset. Like if some images already send to server Bluetooth are deleted from the computer system memory or hard disk, then the end user can use the reset option on the images already sent list, so that to send the image again to server (pc or laptop ) or to save it again in the computer system memory (secondary memory). Client Bluetooth software also provides help for the first time users, which explain that how to use the Client Bluetooth software step by step, shown in figure 7.

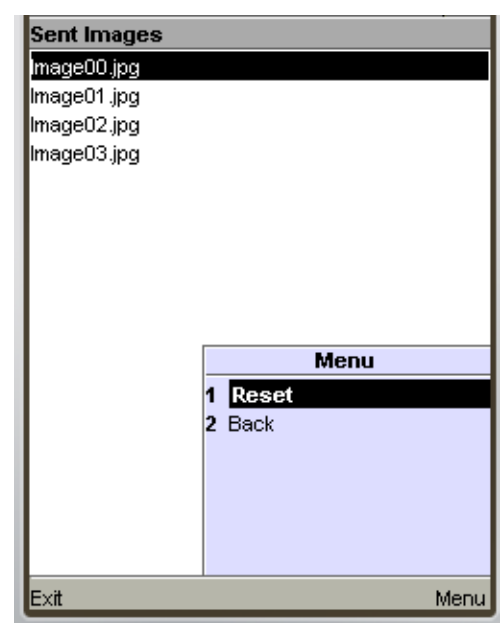

Reset sent images list

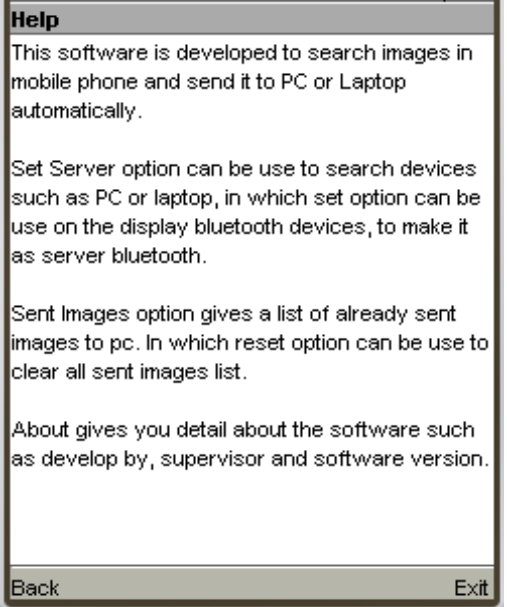

Help screen

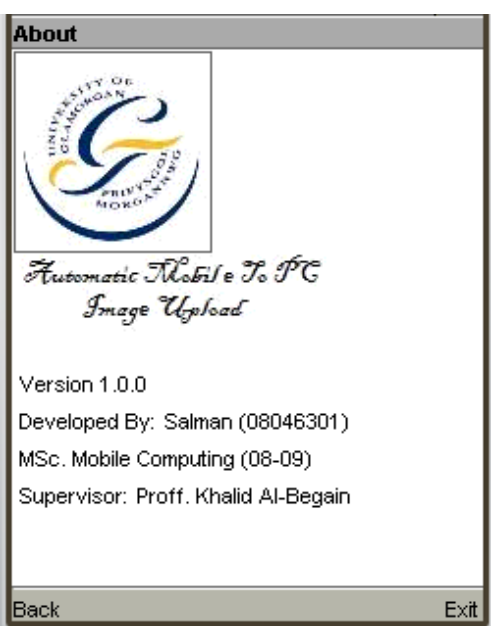

About screen

Figure 7: Reset sent images list, Help screen \& About screen

4. Conclusion: Java is a platform independent, object oriented and multithreaded programming environment. Java is the foundation for network and Web services, applications, robotics, and platform independent desktops and for other embedded devices. One of the best characteristic of Java programming is portability, which means that programs written in the Java language must run on any supported hardware or OS platform.

J2ME (Java 2 Micro Edition) is the Most Ubiquitous Application Platform for Mobile Devices. J2ME (Java 2 Micro Edition) provides a robust flexible environment for all application running on cell phones and also other embedded devices that include cell phones, PDAs (Personal Digital Assistants), printers and also TV set top boxes. J2ME (Java 2 Micro Edition) includes built-in network protocols, robust security, flexible user 
interfaces and support for networked and offline applications that can be downloaded dynamically. Applications based on J2ME (Java 2 Micro Edition) are portable across many devices, yet influence each device's native capabilities.[13][12]

To conclude, Automatic Mobile to PC Images Upload software has two parts client Bluetooth software and server Bluetooth software. Client Bluetooth software is for mobile phone and server Bluetooth software is for PC or Laptop. The major task of client Bluetooth is to search the server Bluetooth software in its Bluetooth range, and once it found that server Bluetooth is in the range, it start searching new images in the mobile phone memory and also in the mobile phone memory card and also check images before sending them to server Bluetooth software so that the images are not sent twice. The major task of the server Bluetooth is receives the images from the client Bluetooth software and then save them in the computer system memory (hard disk). However, Automatic Mobile to PC Images Upload software is not sign or not trusted therefore it asks for permission from user ever time when it read image(s) file from mobile phone memory or from memory card of mobile phone and for Bluetooth connectivity with PC or Laptop. However, if a certificate is provided from trusted third party such as Thawte or VeriSign then Automatic Mobile to PC Images Upload software can work automatically, whenever the mobile phone found the PC or laptop in its Bluetooth range.

\section{REFERENCES}

[1] Bala Kumar, C., Kline, P. J., \& Thompson, T. J. (2004). Example Applications. Bluetooth Application Programming with the Java APIs, 243-318.

[2] Bala Kumar, C., Kline, P. J., \& Thompson, T. J. (2004). Introduction. Bluetooth Application Programming with the Java APIs, 1-22

[3] Bray, J., \& Sturman, C. F. (2000). Bluetooth: Connect without the Cables (1st ed.). United States: Prentice Hall PTR.

[4] Dexter, M. (2007, June 1). Eclipse And Java: Free Video Tutorials. Retrieved 9 October 2014, from http://eclipsetutorial.sourceforge.net/totalbeginner.html

[5] Flanagan, D. (2011). JavaScript: The Definitive Guide: Activate Your Web Pages (6th ed.). United States: O’Reilly Media, Inc, USA.

[6] Gast, M. S. (2005). 802.11 Wireless Networks: The Definitive Guide, Second Edition (Definitive Guide) (2nd ed.). United States: O'Reilly Media, Inc, USA.

[7] Gehrmann, C. (2007). Bluetooth Security. Network Security, 313-329. http://doi.org/10.1002/9780470099742.ch18

[8] Gratton, D. A. (2002). Bluetooth profiles: the definitive guide (1st ed.). London: Prentice Hall PTR.

[9] Hopkins, B., \& Antony, R. (2003). Bluetooth For Java (1st ed.). Berkeley, CA: Apress L. P.

[10] Hopkins, B., \& Antony, R. (2003). Bluetooth for Java. -Infinity. http://doi.org/10.1007/978-1-4302-0763-4

[11] Hopkins, B., \& Antony, R. (2003). Bluetooth with J2ME MIDP. Bluetooth for Java, 75-96. http://doi.org/10.1007/978-1-4302-0763-4_5

[12] Knudsen, J. B. (2003). Wireless Java: Developing with J2ME, Second Edition (Books for Professionals By Professionals) (2nd ed.). Berkeley, CA: Apress.

[13] McNamara, G., \& Yang, Y. (2008). Creating a mobile P2P file sharing environment over Bluetooth. 2008 Third International Conference on Pervasive Computing and Applications. http://doi.org/10.1109/icpca.2008.4783731

[14] Shaw, R. (2003). Wireless networking made easy: everything you need to know to build your own PANs, LANs, and WANs (1st ed.). New York: AMACOM/American Management Association.

[15] Thompson, T. J., Kline, P. J., Kumar, B. C., \& Kumar, C. B. (2008). Bluetooth Application Programming with the Java APIs Essentials Edition (The Morgan Kaufmann Series in Networking) (The Morgan Kaufmann Series in Networking). Amsterdam: Morgan Kaufmann Publishers In.

[16] Mobile Magazine. (n.d.). Retrieved 9 August 2013, from http://www.mobilemag.com 